\title{
Sideways glance: does dietary restriction promote longevity, though impairing fecundity? Not necessarily, if the diet has a correct nutrient balance
}

\author{
Sancia Gaetani · Fabio Virgili
}

Published online: 5 March 2010

(C) Springer-Verlag 2010

A wide body of evidence indicates that genetic and environmental interventions affect lifespan. Nutrition is a key regulator of tissue growth and dietary restriction (DR), the reduction in dietary intake without malnutrition is one of the several interventions that have been shown to increase longevity in invertebrate and vertebrate organisms [1-4]. In fact, DR extends lifespan in invertebrate model organisms such as the budding yeast S.cerevisiae [5], the nematode worm C. elegans [6], the fruit fly Drosophila [7], as well as laboratory rodents, where its effects were first observed. [8]. During DR, lifespan reaches a peak as nutrition is lowered, but with further reduction in nutrients, starvation causes a decline in the lifespan. The invertebrate model organisms are relatively short lived and easy to maintain and manipulate under laboratory conditions; they are therefore an important resource for analysing the mechanisms by which interventions such as DR increase longevity. At least some mechanisms of ageing show a remarkable degree of evolutionary conservation; an understanding of ageing mechanisms in invertebrate model organisms can therefore be very useful in identifying homologous mechanisms in mammals.

During metazoan evolution, the control of cell growth has evolved from a simple cell autonomous response to nutrient levels to a complex network of intercellular growth factor-mediated signals. Despite these layers of control, individual cells of higher eukaryotes have retained the ability to sense and respond directly to levels of nutrients such as amino acids and others [9]. This primal mode of regulation may serve as a checkpoint to gauge the appropriateness of intercellular growth signals, and coordination

S. Gaetani $(\bowtie) \cdot$ F. Virgili

INRAN, Via Ardeatina 546, 00178 Rome, Italy

e-mail: sancia.gaetani@gmail.com of these regulatory inputs is likely to be essential for normal cell growth, proliferation and survival.

"Cell" has published in the issue of October 2, 2009, a paper entitled: "4E-BP extends lifespan upon dietary restriction by enhancing mitochondrial activity in Drosophila" by Zid and coworkers [10], where interesting observations are reported on the mechanisms of lifespan extension and the slowing of age-related diseases upon DR in D. melanogaster. The conservation of signalling pathways and the short lifespan render this organism an excellent model for the investigation. In this paper, the authors suggested possible mechanisms which may prolong lifespan during times of nutrient limitations. It has become apparent in recent years that nutrient-sensing growth pathways are important regulators of lifespan. In order to assess their nutritional status, cells measure the availability of energy and of amino acids. The target of rapamycin (TOR) pathway, a key nutrient-sensing pathway conserved from yeast to humans, integrates nutrient and environmental signals to mediate growth and metabolism. TOR was discovered in 1991 by Hall et al. [11]. The authors identified two genes in yeast, TOR1 and TOR2, by virtue of mutants that conferred resistance to the antifungal drug rapamycin. Subsequent studies led to the identification of a single TOR in other organisms, including mammals [12]. TOR protein kinases promote cell growth and proliferation in response to nutrients and growth factors. The biochemical purification of TOR-associated proteins has revealed that TOR kinase is present in two complexes, with distinct sets of binding partners. Both TOR complexes have important roles in growth control, but they act in different ways. TOR complex 1 (TORC1) is the major rapamycinsensitive form of TOR and is the primary mediator of energy and amino acid sensing for growth control. TOR complex 2 (TORC2) is insensitive to the inhibitory effects 
of rapamycin, and it phosphorylates substrates distinct from those phosphorylated by TORC1. TORC2 is emerging to have a modulatory role in signalling events, such as insulin signalling. The mammalian target of rapamycin [13] (mTOR, also known as FRAP or RAFT1) has been implicated in such coordination. mTOR is a large serine/ threonine kinase $(289 \mathrm{kDa})$ of the phosphoinositide 3-kinase (PI3 K)-related family, conserved throughout the evolution. Inactivation of mTOR by rapamycin results in G1 arrest accompanied by a dephosphorylation of two mTOR's targets, $\mathrm{p} 70^{\mathrm{S} 6 \mathrm{~K}}$ and $4 \mathrm{E}-\mathrm{BP}$. These proteins regulate ribosome biogenesis and cap-dependent translation, respectively. Their phosphorylation is required to achieve the high levels of protein synthesis necessary for cell cycle entry. 4E-BP (initiation factor IF4E binding protein), a protein phosphorylated by TOR, regulates mRNA translation and growth in flies and in mammals [14]. When TOR pathway is inhibited, the hypophosphorylated form of 4E-BP acts as a translational repressor by binding the protein synthesis initiation factor IF4E, blocking the activity of the IF4F complex. The eukaryotic initiation factor 4F (eIF4F) complex mediates growth-dependent protein synthesis. This activity is accomplished by regulating the association of the mRNA cap binding protein eIF4E with the scaffold protein eIF4G, both components of the eIF4F complex. eIF4G helps the assembling of eIF4F complex by bridging the poly(A) binding proteins (PABPs) with eIF4E [15]. This leads to the circularization of mRNAs with a synergistic effect on the rate of translation [16]. Transcripts with extensive secondary structure in their $5^{\prime}$ untranslated regions (UTRs) are very sensitive to the activity of the eIF4F cap binding complex. In cultured mammalian cells, the overexpression of eIF4E causes fibroblasts transformation and cell size increase that can be reversed by increasing the abundance of $4 \mathrm{E}-\mathrm{BP}$. eIF4E is critical for growth in Drosophila, and its upregulation has been observed in some cancers [17]. Finally, its overexpression has been shown to accelerate the senescence in mammalian cells [18]. It has been shown [19, 20] that inhibition of ribosomal genes extend lifespan of C. elegans and $S$. cerevisiae. These findings, and several others in converging to the same direction, suggest an important role of mRNA translation in modulating the ageing process.

Even though the regulation of gene expression at the level of genomewide transcription has led to advances in the field of ageing, the studies on mRNA translation utilized by Zid and coworkers [10] provided new insights into the ageing processes that are modulated by DR in D. melanogaster. The authors separated mRNAs bound to varying number of ribosomes by density gradient centrifugation and made the novel observation that, under DR, some mRNAs are differentially loaded onto ribosomes compared to rich nutrient conditions and mitochondrial electron transport components were one specific class of genes upregulated at translational level upon DR. The inhibition of the expression of mitochondrial electron transport chain (ETC) subunits diminished the DR-dependent lifespan extension, suggesting a key role for the enhancement of mitochondrial function upon DR in lifespan extension. The modulation of mitochondrial function was dependent on d4E-BP and was associated with the structural properties of the $5^{\prime}$ UTR. Furthermore, the DRdependent extension in lifespan required d4E-BP which was also found to be sufficient to extend lifespan.

In order to obtain DR in D. melanogaster, the authors reduced the concentration of yeast extract in the fly diet keeping constant sucrose concentration. This treatment was sufficient to extend lifespan. They then investigated the changes in mRNA translation and observed a decrease in protein synthesis in this experimental condition. The relative translation rate of an mRNA can be inferred from the number of ribosomes it recruits, as initiation is the ratelimiting step for the translation of most mRNAs. The study of the polysomal profiles showed that, under DR, an overall reduction in the number of ribosomes bound to mRNAs and a decrease in ${ }^{35} \mathrm{~S}$-methionine incorporation into proteins were present.

In DR, even though the overall proteins synthesized are decreased, a number of nuclear encoded mitochondrial genes, including those in Complex I and IV of the ETC, showed increased ribosomal loading and enhanced overall activity in this dietary condition. Interestingly, the authors found that various mitochondrial genes possess shorter 5'UTRs, with lower GC content and less secondary structure than the average cell proteins. These characteristics came out to be important for their enhanced mRNA translation. The translational repressor e4E-BP, the eukaryotic translation initiation factor $4 \mathrm{E}$ binding protein, was upregulated upon DR and mediated DR-dependent changes in mitochondrial activity and lifespan extension. The increase in mitochondrial ETC activity was important for the lifespan extension upon DR. This was investigated by reduction in individual mitochondrial ETC Complex I and Complex IV subunits, using RNAi. The RNAi knockdown was sufficient to lower Complex I and IV mRNA levels under different yeast concentrations and to counter the lifespan increase induced by DR. These data demonstrate the importance of the mitochondrial ETC function in the lifespan extension upon DR in Drosophila and presumably also in all the other organisms.

These results imply that translational regulation of nuclear-encoded mitochondrial gene expression by 4E-BP plays an important role in lifespan extension upon DR. It has been suggested recently that an age-related decline in metabolic function as evidenced by reduced expression of genes in the electron transport chain may be a common 
feature among species as diverse as flies, worms and mammals [21, 22]. It is therefore significant the observation that DR which is known to slow the aging in several species, increases the translation of mitochondrial electron transport chain genes via the downstream TOR effector 4EBP. This could have a protective effect by maintaining the function of the electron transport chain and hence ATP production with age. The results also suggest that d4E-BP modulates mRNA translation to induce a metabolic shift towards increased mitochondrial capacity which may prolong lifespan during times of nutrient limitation and translational regulation of nuclear-encoded mitochondrial gene expression by 4E-BP plays an important role in lifespan extension upon DR.

Interestingly, simultaneously to the paper of Zid et al. on October 2, 2009, another paper was published on Science entitled "Ribosomal protein S6 kinase signalling regulates mammalian life span". Dominique J. Withers and coworkers [23], from the Institute of Healthy Ageing, Department of Medicine of University College of London found that the deletion of ribosomal S6 protein kinase 1 (S6K1), a component of the nutrient-responsive mTOR signalling pathway, prolonged life of about 80 days, i.e. $9 \%$ more than control mice, with females surviving $20 \%$ longer. The animals were also less likely to develop certain pathological signs of ageing, such as bone, immune and motor dysfunction and loss of insulin sensitivity. S6K1 deletion induced gene-expression patterns very similar to those observed in mice undergoing long-term caloric restriction or with pharmacological activation of AMPactivated protein kinase (AMPK), suggesting that manipulating S6K1 signalling could be a good strategy to find drugs mimicking the positive effects of DR. S6K1 transduces anabolic signals to regulate cell size, growth and metabolism through various mechanisms that include effects on the translational machinery and on cellular energy levels through the activity of AMPK. These results are in agreement with those described previously [10]. In fact, inactivation of mTOR with rapamycin results in G1 arrest and in a dephosphorylation of two mTOR's targets, 4E-BP investigated by Zid and coworkers [10] and $\mathrm{p} 70^{\mathrm{S} 6 \mathrm{~K}}$ studied by Withers and coworkers [23]. The phosphorylation of both proteins is necessary to achieve a high level of protein synthesis essential for cell cycle entry. This effect would be responsible for influencing lifespan and agerelated pathologies. However, it has also been reported that dietary restriction together with an extension of lifespan is associated to a reduction in fecundity in diverse organisms [24]. The widely accepted interpretation was that DR induces an adaptive reallocation of nutrients from reproduction to essential functions for survival [25] and therefore long life under DR and high fecundity is mutually exclusive through competition for the same limiting nutrients. Very recently, this assumption has been tested and disproved by Grandison et al. in their paper entitled "Amino-acid imbalance explains extension of lifespan by dietary restriction in Drosophila" [26]. The authors reported that this idea is almost certainly wrong and identified the nutrients producing the responses of lifespan and fecundity, respectively, to DR in Drosophila. These authors found that dietary amino acids are responsible for lifespan shortening and increasing reproduction, but both longevity and fecundity can be maximized when intake of these nutrients is finely tuned. The authors have identified the specific nutrients that modulate lifespan and reproduction by manipulating all the components of a defined diet. They fed female flies a restricted diet that extend lifespan at the expense of fecundity and then tried to restore the short-life and high-fecundity characteristics of fully fed flies by adding back specific nutrients. The addition of carbohydrates, lipids or vitamins had no effects. Differently, the addition of amino acids shortened lifespan and reverted egg production to the level observed under full feeding. The authors then asked the question of whether all amino acids contributed equally to this effect. The answer was that all non-essential amino acids (NEAAs) only marginally shortened lifespan and did not affect fecundity whereas the addition of all essential amino acids (EAAs) decreased lifespan and increased egg production, as much as combining all amino acids or full feeding. The authors then manipulated individually each EAA and found that methionine supplementation alone was able to restore fecundity at the level of full feeding, but without reducing lifespan and no single EAA shortened lifespan to the fully fed state. Yet, adding all EAAs except methionine failed to reduce lifespan, suggesting that methionine together with one or several EAAs is responsible for the lifespan-shortening effect of full feeding. This result is in agreement with the results of the work showing that methionine restriction increases fly lifespan [27]. To explain the effect, one possibility is that methionine restriction counters oxidative damage increasing the levels of glutathione [27]. Another explanation might be that methionine restriction extends lifespan by reducing signalling through a major regulator of longevity in many species [25, 27-29], the IIS-insulin/ insulin-like growth factor IGF-pathway. In fact, methionine-deficient mice show lowered levels of IGF-1 pathway [25]. Moreover, when Grandison et al. [26] added back EAAs to the diet, they observed a strong decrease in lifespan in normal flies, but only a very small decrease in mutant flies lacking the insulin-like receptor that mediates the IIS activity. These results demonstrate that the IIS pathway mediates key effects of amino acids on ageing and reproduction. Addition of methionine did not promote fecundity in these mutants. During development and growth, IIS interacts with TOR signalling [10, 29], through 


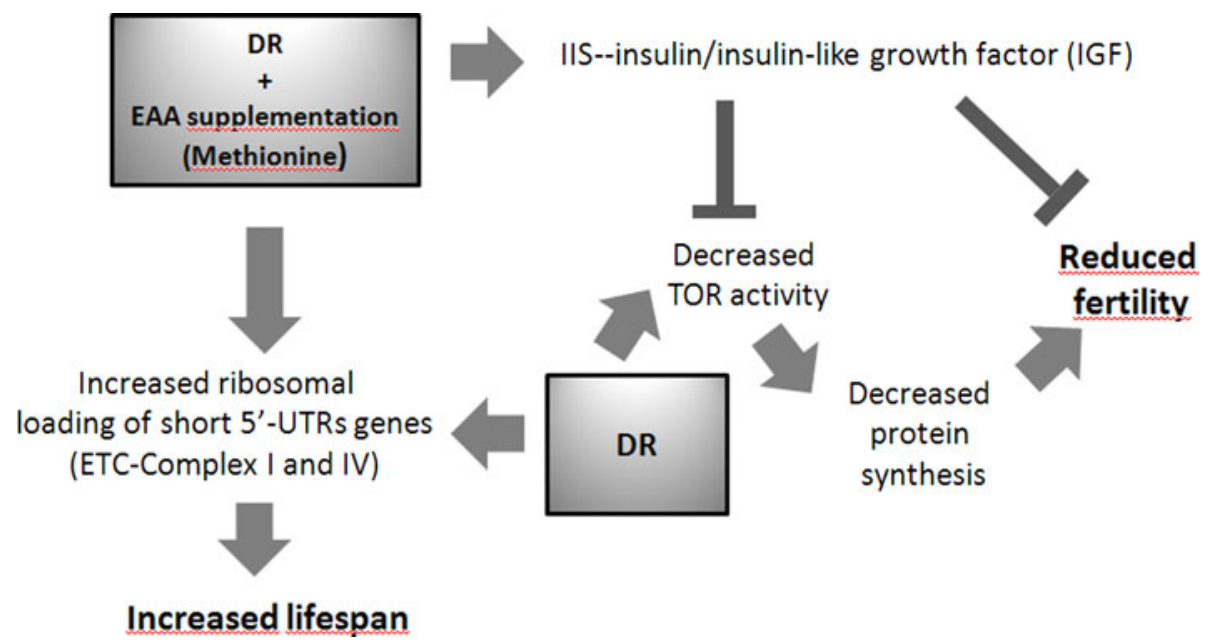

Fig. 1 Dietary restriction (DR) is associated with a decreased activity of the target of rapamycin (TOR) pathway which down regulates the level of protein synthesis, leading to a reduced fertility. DR induces an increase in the ribosomal loading of genes encoding for proteins participating to the electron transfer chain (ETC.) having shorter $5^{\prime}$ UTRs, with lower GC content and lesser secondary structure than the average cell proteins. The increased expression of Complex I and
Complex IV proteins results in an enhanced ETC functions and increased lifespan. Essential amino acid (EAA) supplementation, and in particular by methionine, in the presence of DR is associated with an of IIS-insulin/insulin-like growth factor (IGF) expression which counters the down-regulation of TOR activity and the negative effects on fertility while lifespan extension is maintained

reported by Grandison and coworkers [26] imply that also in mammals, the benefits of DR for health and lifespan may be obtained without impairing fecundity and without DR itself, by a suitable balance of nutrients in the diet.

\section{References}

1. Koubova J, Guarente L (2003) How does calorie restriction work? Genes Dev 17:313-321

2. Zimmerman JA, Malloy V, Krajcik R, Orentreich N (2003) Nutritional control of aging. Exp Gerontol 38:47-52

3. Mastro EJ (2000) Caloric restriction and aging: an update. Exp Gerontol 35:299-305

4. Katewa SD, Kapahi P (2010) Dietary restriction and aging, 2009. Aging cell "accepted article". doi: 10.1111/j.1474-9726.2010. 00552.x

5. Jiang JC, Jaruga E, Repnevskaya MV, Jazwinski SM (2000) An intervention resembling caloric restriction prolongs life span and retards aging in yeast. FASEB J 14:2135-2137

6. Lakowski B, Hekimi S (1998) The genetics of caloric restriction in Caenorhabditis elegans. Proc Natl Acad Sci USA 95:1309113096

7. Chapman T, Partridge L (1996) Female fitness in Drosophila melanogaster: an interaction between the effect of nutrition and of encounter rate with males. Proc R Soc Lond B Biol Sci 263:755-759

8. McCay CM, Cromwell MF, Maynard LA (1935) The effect of retarded growth upon the length of lifespan and upon the ultimate body size. J Nutr 10:63-79

9. Kimball SR, Jefferson LS (2000) Regulation of translation initiation in mammalian cells by amino acids. In: Sonenberg N, Hershey JWB, Mathews MB (eds) Translational control of gene expression. Cold Spring Harbor Laboratory Press, Cold Spring Harbor, NY, pp 561-579 
10. Zid BM, Rogers AN, Katewa SD, Vargas MA, Kolipinski MC, Lu TA, Benzer S, Kapahi P (2009) 4E-BP extends lifespan upon dietary restriction by enhancing mitochondrial activity in Drosophila. Cell 139:149-160

11. Heitman J, Movva NR, Hall MN (1991) Targets for cell cycle arrest by the immunosuppressant rapamycin in yeast. Science 253:905-909

12. Sabatini DM, Erdjument-Bromage H, Lui M, Tempst P, Snyder SH (1994) RAFT1: a mammalian protein that binds to FKBP12 in a rapamycin-dependent fashion and is homologous to yeast TORs. Cell 78:35-43

13. Laplante M, Sabatini DM (2009) mTOR signaling at a glance. J Cell Sci 122:3589-3594

14. Harris TE, Lawrence JC (2003) TOR signaling. Sci. STKE. re 15

15. Sonenberg N, Hershey JWB, Mathews BM (2000) Translational control of gene expression, 2nd edn. Cold Spring Harbor Laboratory Press, Cold Spring Harbor, NY

16. Richter JD, Sonenberg N (2005) Regulation of cap-dependent translation by eIF4E inhibitory proteins. Nature 433:477-480

17. Petroulakis E, Mamane $Y$, Le Bacquer O, Shahbazian D, Sonenberg N (2006) mTOR signaling: implications for cancer and anticancer therapy. Brit J Cancer 94:195-199

18. Ruggero D, Montanaro L, Ma L, Xu W, Londei P, Cordon-Cardo C, Pandolfi PP (2004) The translation factor eIF-4E promotes tumor formation and cooperates with c-Myc in lymphomagenesis. Nat Med 10:484-486

19. Hansen M, Taubert S, Crawford D, Libina N, Lee SJ, Kenyon C (2007) Lifespan extension by conditions that inhibit translation in Caenorhabditis elegans. Aging Cell 6:95-110

20. Steffen KK, MacKay VL, Kerr EO, Tsuchiya M, Hu D, Fox LA, Dang N, Johnston ED, Oakes JA, Tchao BN, Pak DN, Fields S, Kennedy BK, Kaeberlein M (2008) Yeast life span extension by depletion of $60 \mathrm{~S}$ ribosomal subunits is mediated by Gcn4. Cell 133:292-302

21. McCarrol SA, Murphy CT, Zou S, Pletcher SD, Chin CS, Jan YN, Kenyon C, Bargmann CI, Li H (2004) Comparing genomic expression patterns across species identifies shared transcriptional profile in aging. Nat Genet 36:197-204

22. Zahn JM, Sonu R, Vogel H, Crane E, Mazan-Mamczarz K, Rabkin R, Davis RW, Becker KG, Owen AB, Kim SK (2006) Transcriptional profiling of aging in human muscle reveals a common aging signature. PLoS genetics 2:e115

23. Selman C, Tullet JM, Wieser D, Irvine E, Lingard SJ, Choudhury AL, Claret M, Al-Qassab H, Carmignac D, Ramadani $\mathrm{F}$ et al (2009) Ribosomal protein S6 kinase 1 signaling regulates mammalian life span. Science 326:140-144

24. Partridge L, Gems D, Withers DJ (2005) Sex and death: what is the connection? Cell 120:461-472

25. Flatt T, Schmidt PS (2009) Integrating evolutionary and molecular genetics of aging. Biochim Biophys Acta 1790:951-962

26. Grandison RC, Piper MD, Partridge L (2009) Amino-acid imbalance explains extension of lifespan by dietary restriction in Drosophila. Nature 462:1061-1064

27. Troen AM, French EE, Roberts JF, Selhub J, Ordovas JM, Parnell LD, Lai CQT (2007) Lifespan modification by glucose and methionine in Drosophila melanogaster fed a chemically defined diet. Age 29:29-39

28. Miller RA, Buehner G, Chang Y, Harper JM, Sigler R, SmithWheelock M (2005) Methionine-deficient diet extends mouse lifespan, slows immune and lens aging, alters glucose, T4, IGF-I and insulin levels, and increases hepatocyte MIF levels and stress resistance. Aging Cell 4:119-125

29. Mair W, Dillin A (2008) Aging and survival: the genetics of lifespan extension by dietary restriction. Annu Rev Biochem 77:727-754

30. Russell SJ, Kahn CR (2007) Endocrine regulation of ageing. Nature Rev Mol Cell Biol 8:681-691

31. Hietakangas V, Cohen SM (2009) Regulation of tissue growth through nutrient sensing. Annu Rev Genet 43:389-410 